\title{
Commentary \\ Towards goal-directed therapy of hepatorenal syndrome: we have the tools but we need the trials
}

\author{
Rajeshwar P Mookerjee and Rajiv Jalan
}

Liver Failure Group, Institute of Hepatology, 69-75 Chenies Mews, London WC1E 6HX, UK

Corresponding author: Rajiv Jalan, r.jalan@ucl.ac.uk

Published: 19 March 2008

This article is online at http://ccforum.com/content/12/2/119

(c) 2008 BioMed Central Ltd

See related research by Umgelter et al., http://ccforum.com/content/12/1/R4
Critical Care 2008, 12:119 (doi:10.1186/cc6804)

\begin{abstract}
Patients with cirrhosis who develop tense ascites and hepatorenal syndrome have a very high mortality and present a management challenge. Current debate stems from a lack of studies evaluating changes in effective arterial blood volume following paracentesis or targeting fluid replacement with appropriate vascular physiological measures to ensure no paracentesis-related circulatory dysfunction. The study by Umgelter and colleagues addresses a goaldirected approach to fluid management in hepatorenal syndrome and raises several mechanistic questions, the answers to which are likely to improve our understanding of the pathophysiology in hepatorenal syndrome and to guide future management.
\end{abstract}

Decompensated cirrhosis is characterized by severe circulatory derangements including progressive splanchnic vasodilatation and portal hypertension. These derangements result in several of the complications of advanced cirrhosis, such as increasing ascites and hepatorenal syndrome (HRS). The splanchnic vasodilatation in turn results in relative arterial underfilling [1], with consequent activation of the neurohumoral system, leading to vasoconstriction of numerous vascular beds including the liver, the kidney and the brain [2]. The more advanced the disease, the greater the activation of these neurohumoral factors, most maximal in the state of HRS [3]. Management of tense ascites in the context of evolving HRS has been debated with the concern that a large-volume paracentesis may evoke paracentesis-induced circulatory dysfunction despite volume replacement, thereby further potentiating HRS.

The study reported by Umgelter and colleagues in the previous edition of Critical Care describes the single and combined effects on haemodynamics and renal function of plasma expansion with albumin and paracentesis in patients with tense ascites and HRS [4]. Maintenance of the global end-diastolic volume was achieved by invasive monitoring of the central volume. The study's key findings were a demonstration that the cardiac index in HRS patients was fluid responsive despite a normal central venous pressure, and that the reduction in intra-abdominal pressure following paracentesis was associated with an improvement in renal function in the context of fluid substitution guided by assessment of the global end-diastolic volume. In their uncontrolled study, a transpulmonary thermodilution technique (the PiCCO system, Pulsion Medical Systems, AG) facilitated measurement of both cardiac output as well as intrathoracic and pulmonary blood volumes. A subtraction of the pulmonary blood volume from the intrathoracic blood volume enabled an estimate of the 'central' blood volume, referred to as the global end-diastolic volume index (GEDVI).

The authors are to be congratulated on their attention to haemodynamic monitoring, since in cirrhosis a mismatch exists between the capacity of the vascular system and the volume available to fill it; that is, there is a reduced 'effective' arterial blood volume [5]. Given the complexity of interpreting central venous pressure measurements in patients with increased intra-abdominal pressure and marked systemic vasodilatation, measurement of the central blood volume (the pulmonary, cardiac and central arterial tree contributions) as performed by Umgelter and colleagues is likely to reflect the closest estimation to this effective arterial volume [4]. The authors observed a significant early increase in the GEDVI after a $200 \mathrm{ml}$ fluid load of $20 \%$ albumin despite no significant increase in central venous pressure and an actual reduction in the systemic vascular resistance. It is likely that this observation can be explained by a reduction of endogenous vasopressors (with unopposed vasodilatation) as a consequence of improved cardiac function, even though vasopressor activity was not specifically measured in this study. 
Previous studies investigating the effects of an albumin load in Child C cirrhosis [6] and in the treatment of bacterial peritonitis [7] have demonstrated a significant reduction in plasma renin activity following therapy. Moreover, it has been suggested that cirrhotic patients exist in a compensated systemic vasodilated state, with a greater contribution of vasoconstrictors such as noradrenaline, angiotensin II and endothelin-1, to maintain basal vascular tone. [8] The relative change in the balance between vasoconstrictors and vasodilators may also explain the drop in systemic vascular resistance observed in the study by Umgelter et al.

Another important finding of this study is the improvement in creatinine clearance and the fractional extraction of sodium following paracentesis, despite GEDVI subsequently increasing, by adopting a 'goal directed' target for fluid substitution [4]. This finding has significant clinical relevance given the reduction in systemic vascular resistance and the previously held concerns for potential to develop postparacentesis circulatory dysfunction, with an aggravation of the hyperdynamic cirrhotic circulatory state.

Although $40 \mathrm{~g}$ albumin infusion does not expand the central blood volume in patients with advanced Child C cirrhosis, a study by Brinch and colleagues does show a significant improvement in the low effective arterial blood volume in these patients associated with a reduction in plasma renin levels alongside an increase in arterial compliance, which may be important in the prevention of circulatory dysfunction [6]. A not too dissimilar result has been achieved using vasoconstrictors and albumin prior to transjugular intrahepatic portosystemic stent shunt insertion in HRS patients, with improvement in fractional sodium excretion and renal function associated with a reduction in plasma renin levels after 1 year of follow-up [9]. A targeted approach to maintaining effective arterial blood volume through an associated decrease in activation of the renin-angiotensin mechanism will, therefore, have a positive effect on renal dysfunction.

Another factor worthy of note is the recognition that there is enhanced sympathetic activation with advancing liver disease which has been suggested to interfere with renal blood flow autoregulation, causing increasing dependence of the renal blood flow on the renal perfusion pressure [3]. The suggested improvement in renal perfusion pressure observed by Umgelter and colleagues may therefore not only be the effect of reducing the intra-abdominal pressure but may also result from the effects of decreasing sympathetic activation, even though this was not measured in their study.

In summary, the data provided by Umgelter et al. provide important support for a goal-directed approach to fluid management in advanced cirrhosis and HRS, and emphasize that the cardiac index may be fluid responsive in such patients despite normal central venous pressure [4]. More extensive studies, however, are required to further validate the methodology to assess the GEDVI in cirrhotic patients before this measure can be implemented as the standard of care in the management of HRS.

\section{Competing interests}

The authors declare that they have no competing interests.

\section{References}

1. Schrier RW, Arroyo V, Bernardi M, Epstein M, Henriksen JH, Rodes J: Peripheral arterial vasodilation hypothesis: a proposal for the initiation of renal sodium and water retention in cirrhosis [see comments]. Hepatology 1988, 8:1151-1157.

2. Nicholls KM, Shapiro MD, Van Putten VJ, Kluge R, Chung HM, Bichet DG, Schrier RW: Elevated plasma norepinephrine concentrations in decompensated cirrhosis. Association with increased secretion rates, normal clearance rates, and suppressibility by central blood volume expansion. Circ Res 1985, 56:457-461.

3. Stadlbauer VP, Wright GA, Banaji M, Mukhopadhya A, Mookerjee $\mathrm{R}$, Moore K, Jalan R: Relationship between activation of the sympathetic nervous system and renal blood flow autoregulation in cirrhosis. Gastroenterology 2008, 134:111-119.

4. Umgelter A, Reindl W, Wagner K, Franzen M, Stock K, Schmid RM, Huber W: Effects of plasma expansion with albumin and paracentesis on haemodynamics and kidney function in critically ill cirrhotic patients with tense ascites and hepatorenal syndrome: a prospective uncontrolled trial. Crit Care 2008, 12: R4.

5. Moller S, Bendtsen F, Henriksen JH: Determinants of the renin-angiotensin-aldosterone system in cirrhosis with special emphasis on the central blood volume. Scand J Gastroenterol 2006, 41:451-458.

6. Brinch K, Moller S, Bendtsen F, Becker U, Henriksen JH: Plasma volume expansion by albumin in cirrhosis. Relation to blood volume distribution, arterial compliance and severity of disease. J Hepatol 2003, 39:24-31.

7. Fernandez J, Navasa M, Garcia-Pagan JC, G-Abraldes J, Jimenez W, Bosch J, Arroyo V: Effect of intravenous albumin on systemic and hepatic hemodynamics and vasoactive neurohormonal systems in patients with cirrhosis and spontaneous bacterial peritonitis. J Hepato/ 2004, 41:384-390.

8. Helmy A, Jalan R, Newby DE, Johnston NR, Hayes PC, Webb DJ: Altered peripheral vascular responses to exogenous and endogenous endothelin-1 in patients with well-compensated cirrhosis. Hepatology 2001, 33:826-831.

9. Wong F, Pantea L, Sniderman K: Midodrine, octreotide, albumin, and TIPS in selected patients with cirrhosis and type 1 hepatorenal syndrome. Hepatology 2004, 40:55-64. 